\title{
A Criterion for the Asymptotic Stability of Singular Differential Systems by the Linear Diagonal Approximation
}

\author{
S. G. Krasovskii \\ Institute for Mathematics, National Academy of Sciences, Minsk, Belarus \\ Received January 5, 2006
}

DOI: $10.1134 /$ S0012266106080040

Consider the singular linear system

$$
\varepsilon \dot{x}=A(t) x, \quad x \in \mathbb{R}^{n}, \quad t \geq 0,
$$

with bounded continuous coefficient matrix $A(t)$ and a small positive parameter $\varepsilon$ multiplying the derivative and the perturbed singular system

$$
\varepsilon \dot{y}=A(t) y+Q(t) y, \quad y \in \mathbb{R}^{n}, \quad t \geq 0, \quad\left(1_{(A+Q) / \varepsilon}\right)
$$

with piecewise continuous perturbation $Q(t),\|Q(t)\| \leq \delta, t \geq 0$.

Starting from the fundamental papers by Tikhonov, numerous papers by Butuzov, Vasil'eva, Fedoryuk, Lomov, Rozov, Mishchenko, Vazov, Shishkin, et al. dealt with the analysis of singularly perturbed systems of a more general form.

Necessary and sufficient conditions for all solutions $y\left(t, y_{0}, \varepsilon\right), y\left(0, y_{0}, \varepsilon\right)=y_{0} \in \mathbb{R}^{n}$, of system $\left(1_{(A+Q) / \varepsilon}\right)$ with continuous matrix $A(t)=\operatorname{diag}\left[a_{1}(t), \ldots, a_{n}(t)\right]$ and with all possible perturbations $Q(t)$ of sufficiently small norm to tend to zero as $\varepsilon \rightarrow+0$ (for fixed $t$ ) on any finite interval $\left[t_{0}, t_{1}\right]$ of the positive half-line not containing the initial time were obtained in [1]. These conditions are as follows: $\int_{0}^{t} \max _{i}\left\{a_{i}(\tau)\right\} d \tau<0, i=1, \ldots, n$, for all $t \in\left[t_{0}, t_{1}\right] \subset\left(0, t_{1}\right]$.

In the present paper, similar conditions are obtained for an arbitrary infinite interval $\left[t_{0},+\infty\right) \subset$ $(0,+\infty)$. Note that, in this case, the condition $\int_{0}^{t} \max _{i}\left\{a_{i}(\tau)\right\} d \tau<0, t \in\left[t_{0},+\infty\right)$, does not guarantee that $\left\|y\left(t, y_{0}, \varepsilon\right)\right\| \rightarrow 0$ as $\varepsilon \rightarrow+0$ for arbitrary perturbations of sufficiently small norm on the entire interval $(0,+\infty)$. Let us illustrate this by an example.

Example. For all solutions $x\left(t, x_{0}, \varepsilon\right)$ of the scalar equation

$$
\varepsilon \dot{x}=-(t+1)^{-1} x, \quad x \in \mathbb{R}, \quad \varepsilon \in(0,1], \quad t \geq 0,
$$

we have $\left|x\left(t, x_{0}, \varepsilon\right)\right|=\left|x_{0}\right| \exp \left[-\varepsilon^{-1} \ln (t+1)\right] \rightarrow 0$ as $\varepsilon \rightarrow+0$ for all $t \in\left[t_{0},+\infty\right) \subset(0,+\infty)$ and for an arbitrary $x_{0} \in \mathbb{R}$. But if we consider the singularly perturbed equation

$$
\varepsilon \dot{y}=-(t+1)^{-1} y+\delta y, \quad y \in \mathbb{R}, \quad \varepsilon \in(0,1], \quad \delta>0, \quad t \geq 0,
$$

whose solutions have the form $y\left(t, x_{0}, \varepsilon\right)=y_{0} \exp \left[\varepsilon^{-1}(\delta t-\ln (t+1))\right]$, then for an arbitrarily small $\delta>0$, there exists a sufficiently large time $T=T(\delta)$, determined by the relation $\delta T \geq \ln (T+1)$, such that $\left|y\left(t, y_{0}, \varepsilon\right)\right| \rightarrow+\infty$ as $\varepsilon \rightarrow+0$ for all $t>T$.

Theorem. The solutions $y\left(t, y_{0}, \varepsilon\right)$ of the linear system $\left(1_{(A+Q) / \varepsilon}\right)$ with a continuous matrix $A(t)=\operatorname{diag}\left[a_{1}(t), \ldots, a_{n}(t)\right]$ satisfy the relation $\lim _{\varepsilon \rightarrow+0} y\left(t, y_{0}, \varepsilon\right)=0$ (uniformly with respect to $\left.t \in\left[t_{0},+\infty\right) \subset(0,+\infty)\right)$ for all $y_{0}=y\left(0, y_{0}, \varepsilon\right) \in \mathbb{R}^{n}$ and for arbitrary piecewise continuous 
matrices $Q(t)$ satisfying the condition $\|Q(t)\| \leq \delta, t \geq 0$, with a sufficiently small number $\delta>0$ if and only if there exists a number $\delta_{0}>0$ such that

$$
\int_{0}^{t}\left[\delta_{0}+\max _{i}\left\{a_{i}(\tau)\right\}\right] d \tau<0, \quad i=1, \ldots, n, \quad \forall t \in\left[t_{0},+\infty\right) .
$$

Proof. Necessity. Consider the upper [2, p. 116] function $r(t)$ given by the relation

$$
r(t) \equiv \max _{i}\left\{a_{i}(t)\right\}, \quad i=1, \ldots, n, \quad t \in[0,+\infty),
$$

and suppose the contrary: for an arbitrarily small $\delta>0$, there exists a point $\eta \equiv \eta(\delta) \in\left[t_{0},+\infty\right)$ such that $\int_{0}^{\eta}[(\delta / 8)+r(\tau)] d \tau \geq 0$, or, with the notation $J(\tau, t) \equiv \int_{\tau}^{t} r(\xi) d \xi[1],(\delta / 8) \eta+J(0, \eta) \geq 0$.

Under this assumption, we prove the existence of an initial vector $y_{0} \in \mathbb{R}^{n}$ and a piecewise continuous perturbation $Q(\cdot),\|Q(\cdot)\| \leq \delta$, such that $\varlimsup_{\varepsilon \rightarrow+0}\left\|y\left(\eta, y_{0}, \varepsilon\right)\right\|>0$ at the above-mentioned point $\eta$.

We take an arbitrary $\delta>0$. On the interval $[0, \eta]$, the functions $r(t)$ and $a_{i}(t), i=1, \ldots, n$, are continuous and hence uniformly continuous; ${ }^{1}$ i.e.,

$$
\begin{aligned}
& \exists T \in(0, \eta]: \quad\left|t^{\prime}-t^{\prime \prime}\right| \leq T \Rightarrow\left|r\left(t^{\prime}\right)-r\left(t^{\prime \prime}\right)\right| \leq \delta / 4, \\
& \left|a_{i}\left(t^{\prime}\right)-a_{i}\left(t^{\prime \prime}\right)\right| \leq \delta / 4, \quad i=1, \ldots, n, \quad \forall t^{\prime}, t^{\prime \prime} \in[0, \eta] .
\end{aligned}
$$

We also require that a closed interval of length $T$ fits an integer number $s$ of times in the closed interval $[0, \eta]$. For this purpose, we reduce $T$ (if necessary) by taking, say, the quantity $\eta /([\eta / T]+1)$ instead of $T$. Here (and only here) [.] is the integer part of a real number.

We perform the partition of the closed interval $[0, \eta]$ by the points $\tau_{k} \equiv k T, \tau_{s} \equiv \eta, k=0, \ldots, s$. For the numbers $k=0, \ldots, s$, we introduce the index $l(k) \in\{1, \ldots, n\}$ equal to the number of a function $a_{i}(t), i \in\{1, \ldots, n\}$, taking the value $r\left(\tau_{k}\right)$ at the point $t=\tau_{k}: a_{l(k)}\left(\tau_{k}\right)=r\left(\tau_{k}\right)$. If there are several functions with this property, then, to be definite, we choose the least of their numbers. [One can indicate algorithms for choosing the index $l(k)$ of several possible variants so as to minimize the number of rotations to be used below in the construction of the perturbation matrix $Q(\cdot)$.]

It follows from the well-known mean-value theorem for an integral of a continuous function [4, p. 113] that for each $k=0, \ldots, s-1$, there exist points $t_{k} \in\left[\tau_{k}, \tau_{k+1}\right]$ with the values $r\left(t_{k}\right) \equiv f_{k}$, $k=0, \ldots, s-1$, of the function $r(t)$ such that

$$
J\left(\tau_{k}, \tau_{k+1}\right)=f_{k} T
$$

moreover, by virtue of the relation $a_{l(k)}\left(\tau_{k}\right)=r\left(\tau_{k}\right)$ and the uniform continuity (3), we have

$$
\left|a_{l(k)}(t)-f_{k}\right| \leq \delta / 2, \quad\left|a_{l(k+1)}(t)-f_{k}\right| \leq \delta / 2, \quad t \in\left[\tau_{k}, \tau_{k+1}\right]
$$

Indeed,

$$
\begin{aligned}
\left|a_{l(k)}(t)-f_{k}\right| & \leq\left|a_{l(k)}(t)-a_{l(k)}\left(\tau_{k}\right)\right|+\left|a_{l(k)}\left(\tau_{k}\right)-f_{k}\right| \\
& \leq\left|a_{l(k)}(t)-a_{l(k)}\left(\tau_{k}\right)\right|+\left|r\left(\tau_{k}\right)-r\left(t_{k}\right)\right| \leq \delta / 4+\delta / 4=\delta / 2, \\
\left|a_{l(k+1)}(t)-f_{k}\right| & \leq\left|a_{l(k+1)}(t)-a_{l(k+1)}\left(\tau_{k+1}\right)\right|+\left|a_{l(k+1)}\left(\tau_{k+1}\right)-f_{k}\right| \\
& \leq\left|a_{l(k+1)}(t)-a_{l(k+1)}\left(\tau_{k+1}\right)\right|+\left|r\left(\tau_{k+1}\right)-r\left(t_{k}\right)\right| \leq \delta / 4+\delta / 4=\delta / 2 .
\end{aligned}
$$

Let us construct the perturbation matrix $Q(\cdot)$.

\footnotetext{
$\overline{{ }^{1} \text { By the definition of }}$ uniform continuity in [3], for each of the functions $a_{i}(t), i=1, \ldots, n$ [and for the function $\left.r(t)\right]$, there exists its own value $T_{i}$ (respectively, $T_{r}$ ). But, by setting $T=\min \left\{T_{i}, T_{r}\right\}, i=1, \ldots, n$, we find that inequalities (3) are valid simultaneously for all considered functions.
} 
1. On the intervals $\left[\tau_{k}, \tau_{k+1}\right)$ on which the indices $l(k)$ and $l(k+1), k \in\{0,1, \ldots, s-1\}$, do not coincide, the perturbation matrix $Q(t)=\left[q_{i j}(t)\right]_{1}^{n}$ has the entries

$$
q_{l(k+j) l(k+j)}(t)=f_{k}-a_{l(k+j)}(t)+(\delta / 8), \quad j=0,1, \quad q_{l(k+1) l(k)}(t)=-q_{l(k) l(k+1)}(t)=\delta / 8,
$$

and all the remaining entries vanish. The norm of this matrix admits the representation

$$
\begin{aligned}
\|Q(t)\|= & \max _{\left(x_{1}^{2}+\cdots+x_{n}^{2}\right)^{1 / 2}=1}\left[\left(q_{l(k) l(k)}(t) x_{l(k)}-(\delta / 8) x_{l(k+1)}\right)^{2}\right. \\
& \left.+\left(q_{l(k+1) l(k+1)}(t) x_{l(k+1)}+(\delta / 8) x_{l(k)}\right)^{2}\right]^{1 / 2} .
\end{aligned}
$$

Let us now estimate this norm from above with the use of the inequalities

$$
\left|q_{l(k+j) l(k+j)}(t)\right| \leq\left|f_{k}-a_{l(k+j)}(t)\right|+\delta / 8 \leq \delta / 2+\delta / 8=5 \delta / 8, \quad j=0,1,
$$

which follow from (5), and the inequalities $x_{l(k)}^{2}+x_{l(k+1)}^{2} \leq 1$ and $\left|x_{l(k)} x_{l(k+1)}\right| \leq 1 / 2$ :

$$
\begin{aligned}
\|Q(t)\| \leq & \max _{\left(x_{1}^{2}+\cdots+x_{n}^{2}\right)^{1 / 2}=1}\left(q_{l(k) l(k)}^{2}(t) x_{l(k)}^{2}+\left|\frac{\delta}{4} q_{l(k) l(k)}(t)\right|\left|x_{l(k)} x_{l(k+1)}\right|+\frac{\delta^{2}}{64} x_{l(k+1)}^{2}\right. \\
& \left.+q_{l(k+1) l(k+1)}^{2}(t) x_{l(k+1)}^{2}+\left|\frac{\delta}{4} q_{l(k+1) l(k+1)}(t)\right|\left|x_{l(k)} x_{l(k+1)}\right|+\frac{\delta^{2}}{64} x_{l(k)}^{2}\right)^{1 / 2} \\
\leq & \max _{\left(x_{1}^{2}+\cdots+x_{n}^{2}\right)^{1 / 2}=1}\left(2\left[\frac{25 \delta^{2}}{64}\left(x_{l(k)}^{2}+x_{l(k+1)}^{2}\right)+\frac{5 \delta^{2}}{32}\left|x_{l(k)} x_{l(k+1)}\right|\right]\right)^{1 / 2} \\
\leq & \left(2\left[\frac{25 \delta^{2}}{64}+\frac{5 \delta^{2}}{64}\right]\right)^{1 / 2}<\delta .
\end{aligned}
$$

2. On the remaining intervals $\left[\tau_{k}, \tau_{k+1}\right), k \in\{0,1, \ldots, s-1\}$, with $l(k)=l(k+1)$, we set $q_{l(k) l(k)}=r(t)-a_{l(k)}(t)+\delta / 8$ in the matrix $Q(t)$, and the remaining entries are set to zero. In this case, by (3), we obtain the norm estimate

$$
\begin{aligned}
\|Q(t)\| & =\left|r(t)-a_{l(k)}(t)+\frac{\delta}{8}\right| \leq\left|r(t)-a_{l(k)}\left(\tau_{k}\right)\right|+\left|a_{l(k)}\left(\tau_{k}\right)-a_{l(k)}(t)\right|+\frac{\delta}{8} \\
& =\left|r(t)-r\left(\tau_{k}\right)\right|+\left|a_{l(k)}\left(\tau_{k}\right)-a_{l(k)}(t)\right|+\frac{\delta}{8} \leq \frac{\delta}{4}+\frac{\delta}{4}+\frac{\delta}{8}=\frac{5}{8} \delta, \quad t \in\left[\tau_{k}, \tau_{k+1}\right) .
\end{aligned}
$$

Therefore, the norm of the perturbation matrix can be estimated as $\|Q(t)\|<\delta, t \in[0, \eta)$.

We take the initial vector $y_{0}=(0, \ldots, 0,1,0, \ldots, 0)$ whose unique nonzero coordinate is at the $l(0)$ th position and consider the sequence of systems $\left(1_{(A+Q) / \varepsilon}\right)$ with values

$$
\varepsilon=\varepsilon_{m} \equiv \delta T \pi^{-1} /(16 m+4), \quad m \in \mathbb{Z}_{+},
$$

of the small parameter. On the interval $[0, \eta]$, we construct a solution $y\left(t, y_{0}, \varepsilon_{m}\right)$ of system $\left(1_{(A+Q) / \varepsilon_{m}}\right)$ with the parameter $\varepsilon_{m}$ and the above-mentioned matrix $Q(\cdot)$ and show that it has only one nonzero coordinate at the times $t=\tau_{k}, k=0, \ldots, s$.

On the intervals $\left[\tau_{k}, \tau_{k+1}\right), k \in\{0,1, \ldots, s-1\}$, with $l(k) \neq l(k+1)$, we perform the rotation of solutions of system $\left(1_{(A+Q) / \varepsilon_{m}}\right)$ with the perturbation matrix $Q(\cdot)$ of type 1 . The Cauchy matrix $Y\left(t, \tau_{k}\right)=\left[x_{i j}\left(t, \tau_{k}\right)\right]_{1}^{n}, t \in\left[\tau_{k}, \tau_{k+1}\right]$, of this system has the entries

$$
\begin{aligned}
x_{l(k) l(k)}\left(t, \tau_{k}\right) & =x_{l(k+1) l(k+1)}\left(t, \tau_{k}\right)=F_{k, m}(t) \cos \left(\frac{\delta}{8 \varepsilon_{m}}\left(t-\tau_{k}\right)\right), \\
x_{l(k+1) l(k)}\left(t, \tau_{k}\right) & =-x_{l(k) l(k+1)}\left(t, \tau_{k}\right)=F_{k, m}(t) \sin \left(\frac{\delta}{8 \varepsilon_{m}}\left(t-\tau_{k}\right)\right), \\
x_{i i}\left(t, \tau_{k}\right) & =\exp \left[\varepsilon_{m}^{-1} \int_{\tau_{k}}^{t} a_{i}(\tau) d \tau\right], \quad i \in\{1, \ldots, n\} \backslash\{l(k), l(k+1)\},
\end{aligned}
$$

DIFFERENTIAL EQUATIONS $\quad$ Vol. 42 No. 82006 
and all remaining entries are zero. (Here and throughout the following, we use the notation $\left.F_{k, m}(t)=\exp \left[\varepsilon_{m}^{-1}\left(f_{k}+\delta / 8\right)\left(t-\tau_{k}\right)\right].\right)$

Therefore, we obtain the representation

$$
Y\left(t, \tau_{k}\right)=\left(\begin{array}{ccccc}
C_{1}\left(t, \tau_{k}\right) & O_{p_{1}(k), 1} & O_{p_{1}(k), p_{2}(k)} & O_{p_{1}(k), 1} & O_{p_{1}(k), p_{3}(k)} \\
O_{1, p_{1}(k)} & F_{k, m}(t) \cos \frac{\delta\left(t-\tau_{k}\right)}{8 \varepsilon_{m}} & O_{1, p_{2}(k)} & -F_{k, m}(t) \sin \frac{\delta\left(t-\tau_{k}\right)}{8 \varepsilon_{m}} & O_{1, p_{3}(k)} \\
O_{p_{2}(k), p_{1}(k)} & O_{p_{2}(k), 1} & C_{2}\left(t, \tau_{k}\right) & O_{p_{2}(k), 1} & O_{p_{2}(k), p_{3}(k)} \\
O_{1, p_{1}(k)} & F_{k, m}(t) \sin \frac{\delta\left(t-\tau_{k}\right)}{8 \varepsilon_{m}} & O_{1, p_{2}(k)} & F_{k, m}(t) \cos \frac{\delta\left(t-\tau_{k}\right)}{8 \varepsilon_{m}} & O_{1, p_{3}(k)} \\
O_{p_{3}(k), p_{1}(k)} & O_{p_{3}(k), 1} & O_{p_{3}(k), p_{2}(k)} & O_{p_{3}(k), 1} & C_{3}\left(t, \tau_{k}\right)
\end{array}\right)
$$

[to be definite, in this representation, we assume that $l(k)<l(k+1)$ ] with the diagonal blocks

$$
\begin{aligned}
& C_{1}\left(t, \tau_{k}\right) \equiv \operatorname{diag}\left\{\exp \left[\frac{1}{\varepsilon_{m}} \int_{\tau_{k}}^{t} a_{1}(\tau) d \tau\right], \ldots, \exp \left[\frac{1}{\varepsilon_{m}} \int_{\tau_{k}}^{t} a_{l(k)-1}(\tau) d \tau\right]\right\}, \\
& C_{2}\left(t, \tau_{k}\right) \equiv \operatorname{diag}\left\{\exp \left[\frac{1}{\varepsilon_{m}} \int_{\tau_{k}}^{t} a_{l(k)+1}(\tau) d \tau\right], \ldots, \exp \left[\frac{1}{\varepsilon_{m}} \int_{\tau_{k}}^{t} a_{l(k+1)-1}(\tau) d \tau\right]\right\}, \\
& C_{3}\left(t, \tau_{k}\right) \equiv \operatorname{diag}\left\{\exp \left[\frac{1}{\varepsilon_{m}} \int_{\tau_{k}}^{t} a_{l(k+1)+1}(\tau) d \tau\right], \ldots, \exp \left[\frac{1}{\varepsilon_{m}} \int_{\tau_{k}}^{t} a_{n}(\tau) d \tau\right]\right\}
\end{aligned}
$$

and with the zero blocks $O_{i, j}, i, j \in\{0,1, \ldots, n-2\}$, containing $i$ rows and $j$ columns, where $p_{1}(k) \equiv l(k)-1, p_{2}(k) \equiv l(k+1)-l(k)-1$, and $p_{3}(k) \equiv n-l(k+1)$.

By (4), at the endpoints $t=\tau_{k+1}$ of the considered intervals, we obtain the entries

$$
\begin{aligned}
x_{l(k) l(k)}\left(\tau_{k+1}, \tau_{k}\right) & =x_{l(k+1) l(k+1)}\left(\tau_{k+1}, \tau_{k}\right)=\exp \left[\frac{8 J\left(\tau_{k}, \tau_{k+1}\right)+\delta T}{8 \varepsilon_{m}}\right] \cos \left(\frac{\delta T}{8 \varepsilon_{m}}\right), \\
x_{l(k+1) l(k)}\left(\tau_{k+1}, \tau_{k}\right) & =-x_{l(k) l(k+1)}\left(\tau_{k+1}, \tau_{k}\right)=\exp \left[\frac{8 J\left(\tau_{k}, \tau_{k+1}\right)+\delta T}{8 \varepsilon_{m}}\right] \sin \left(\frac{\delta T}{8 \varepsilon_{m}}\right) .
\end{aligned}
$$

For the above-mentioned values $\varepsilon_{m}=\delta T \pi^{-1} /(16 m+4), m \in \mathbb{Z}_{+}$, we find that the only nonzero entries in the Cauchy matrix $Y\left(\tau_{k+1}, \tau_{k}\right)$ are

$$
\begin{aligned}
x_{l(k+1) l(k)}\left(\tau_{k+1}, \tau_{k}\right) & =-x_{l(k) l(k+1)}\left(\tau_{k+1}, \tau_{k}\right)=\exp \left[\frac{8 J\left(\tau_{k}, \tau_{k+1}\right)+\delta T}{8 \varepsilon_{m}}\right], \\
x_{i i}\left(\tau_{k+1}, \tau_{k}\right) & =\exp \left[\frac{1}{\varepsilon_{m}} \int_{\tau_{k}}^{\tau_{k+1}} a_{i}(\tau) d \tau\right], \quad i \in\{1, \ldots, n\} \backslash\{l(k), l(k+1)\} .
\end{aligned}
$$

Therefore, on the intervals $\left[\tau_{k}, \tau_{k+1}\right]$ on which $l(k) \neq l(k+1)$, we rotate the solutions of the system $\left(1_{(A+Q) / \varepsilon_{m}}\right)$ by the angle $(\pi / 2)+2 \pi m$ from the axis $O y_{l(k)}$ towards the axis $O y_{l(k+1)}$ in the $n$-dimensional space with the simultaneous change of the norm of the solution being constructed. Since the value $y\left(\tau_{k}, y_{0}, \varepsilon_{m}\right)$ has only the $l(k)$ th nonzero coordinate, it follows that, after the rotation, the value $y\left(\tau_{k+1}, y_{0}, \varepsilon_{m}\right)$ has only the $l(k+1)$ th nonzero coordinate, and the norm of such a solution admits the representation

$$
\left\|y\left(\tau_{k+1}, y_{0}, \varepsilon_{m}\right)\right\|=\exp \left[\frac{8 J\left(\tau_{k}, \tau_{k+1}\right)+\delta T}{8 \varepsilon_{m}}\right]\left\|y\left(\tau_{k}, y_{0}, \varepsilon_{m}\right)\right\|
$$

On the other hand, the norm of the Cauchy matrix $\left\|Y\left(\tau_{k+1}, \tau_{k}\right)\right\|$ is equal to

$$
\exp \left[\varepsilon^{-1}\left(J\left(\tau_{k}, \tau_{k+1}\right)+\delta T / 8\right)\right]
$$


which follows from the upper bounds

$$
\begin{aligned}
\left\|Y\left(\tau_{k+1}, \tau_{k}\right)\right\|= & \max _{\left(x_{1}^{2}+\cdots+x_{n}^{2}\right)^{1 / 2}=1}\left[\sum_{\substack{i=1 \\
i \neq l(k), l(k+1)}}^{n}\left(\exp \left[\frac{2}{\varepsilon_{m}} \int_{\tau_{k}}^{\tau_{k+1}} a_{i}(\tau) d \tau\right] x_{i}^{2}\right)\right. \\
& \left.+\exp \left[\frac{8 J\left(\tau_{k}, \tau_{k+1}\right)+\delta T}{4 \varepsilon_{m}}\right]\left(x_{l(k)}^{2}+x_{l(k+1)}^{2}\right)\right]^{1 / 2} \\
\leq & \max _{\left(x_{1}^{2}+\cdots+x_{n}^{2}\right)^{1 / 2}=1}\left[\exp \left[\frac{8 J\left(\tau_{k}, \tau_{k+1}\right)+\delta T}{4 \varepsilon_{m}}\right]\left(x_{1}^{2}+\cdots+x_{n}^{2}\right)\right]^{1 / 2} \\
= & \exp \left[\frac{8 J\left(\tau_{k}, \tau_{k+1}\right)+\delta T}{8 \varepsilon_{m}}\right]
\end{aligned}
$$

and the lower bounds

$$
\left\|Y\left(\tau_{k+1}, \tau_{k}\right)\right\| \geq\left|x_{l(k+1) l(k)}\left(\tau_{k+1}, \tau_{k}\right)\right|=\exp \left[\frac{8 J\left(\tau_{k}, \tau_{k+1}\right)+\delta T}{8 \varepsilon_{m}}\right]
$$

for this norm. Therefore, we obtain the relation

$$
\left\|y\left(\tau_{k+1}, y_{0}, \varepsilon_{m}\right)\right\|=\left\|Y\left(\tau_{k+1}, \tau_{k}\right)\right\|\left\|y\left(\tau_{k}, y_{0}, \varepsilon_{m}\right)\right\| .
$$

This is the maximum [5] solution on the interval $\left[\tau_{k}, \tau_{k+1}\right]$; moreover, the vector $y\left(\tau_{k+1}, y_{0}, \varepsilon_{m}\right)$ has the unique nonzero $l(k+1)$ th coordinate, whose growth on the next interval is maximal; therefore, this coordinate remains maximal on the next interval as well.

On the remaining intervals $\left[\tau_{k}, \tau_{k+1}\right), k \in\{0,1, \ldots, s-1\}$, at whose endpoints the indices $l(k)$ and $l(k+1)$ coincide, we use a perturbation matrix $Q(\cdot)$ of type 2 . In this case, system $\left(1_{(A+Q) / \varepsilon_{m}}\right)$ acquires the form

$$
\varepsilon_{m} \dot{y}=\operatorname{diag}\left[a_{1}(t), \ldots, a_{l(k)-1}(t), r(t)+\delta / 8, a_{l(k)+1}(t), \ldots, a_{n}(t)\right] y,
$$

and its Cauchy matrix is

$$
\begin{aligned}
Y\left(t, \tau_{k}\right)=\exp \left\{\frac { 1 } { \varepsilon _ { m } } \operatorname { d i a g } \left[\int_{\tau_{k}}^{t} a_{1}(\tau) d \tau, \ldots, \int_{\tau_{k}}^{t} a_{l(k)-1}(\tau) d \tau, J\left(\tau_{k}, t\right)+\frac{\delta}{8}\left(t-\tau_{k}\right),\right.\right. \\
\\
\left.\left.\int_{\tau_{k}}^{t} a_{l(k)+1}(\tau) d \tau, \ldots, \int_{0}^{t} a_{n}(\tau) d \tau\right]\right\}
\end{aligned}
$$

with the norm

$$
\left\|Y\left(t, \tau_{k}\right)\right\|=\exp \left[\varepsilon^{-1}\left(J\left(\tau_{k}, t\right)+\delta\left(t-\tau_{k}\right) / 8\right)\right] .
$$

Since, as was shown above, the vector $y\left(\tau_{k}, y_{0}, \varepsilon_{m}\right)$ has the only nonzero $l(k)$ th coordinate [or, which is the same, the $l(k+1)$ th coordinate], it follows that at the time $t=\tau_{k+1}$, the norm of the solution $y\left(t, y_{0}, \varepsilon_{m}\right)$ admits the representation

$$
\begin{aligned}
\left\|y\left(\tau_{k+1}, y_{0}, \varepsilon_{m}\right)\right\| & =\exp \left[\varepsilon^{-1}\left(J\left(\tau_{k}, \tau_{k+1}\right)+\delta T / 8\right)\right]\left\|y\left(\tau_{k}, y_{0}, \varepsilon_{m}\right)\right\| \\
& =\left\|Y\left(\tau_{k+1}, \tau_{k}\right)\right\|\left\|y\left(\tau_{k}, y_{0}, \varepsilon_{m}\right)\right\| .
\end{aligned}
$$

Therefore, the norm of the solution $y\left(t, y_{0}, \varepsilon_{m}\right)$ of system $\left(1_{(A+Q) / \varepsilon}\right)$ (which is a maximum solution on each of the intervals $\left.\left[\tau_{k}, \tau_{k+1}\right], k=0, \ldots, s-1\right)$ satisfies the relations

$$
\begin{aligned}
\left\|y\left(\eta, y_{0}, \varepsilon_{m}\right)\right\| & =\left\|y_{0}\right\| \prod_{k=0}^{s-1}\left\|Y\left(\tau_{k+1}, \tau_{k}\right)\right\|=\left\|y_{0}\right\| \exp \left[\sum_{k=0}^{s-1} \frac{8 J\left(\tau_{k}, \tau_{k+1}\right)+\delta T}{8 \varepsilon_{m}}\right] \\
& =\left\|y_{0}\right\| \exp \left[\frac{8 J(0, \eta)+\delta \eta}{8 \varepsilon_{m}}\right] .
\end{aligned}
$$

DIFFERENTIAL EQUATIONS $\quad$ Vol. 42 No. 82006 
By the assumption stipulated at the beginning of the proof, we have $(\delta / 8) \eta+J(0, \eta) \geq 0$. Therefore, in the limit as $\varepsilon_{m} \rightarrow 0$, we obtain

$$
\lim _{m \rightarrow \infty}\left\|y\left(\eta, y_{0}, \varepsilon_{m}\right)\right\| \geq\left\|y_{0}\right\|>0
$$

and this contradicts the relation $\lim _{\varepsilon \rightarrow+0} y\left(t, y_{0}, \varepsilon\right)=0$ for all $t \in\left[t_{0},+\infty\right)$ from the assumptions of the theorem. The obtained contradiction implies the necessity of the assumptions of the theorem.

Sufficiency. We use the estimate

$$
\left\|y\left(t, y_{0}, \varepsilon\right)\right\| \leq\left\|y_{0}\right\| \exp \left\{\varepsilon^{-1}[\delta t+J(0, t)]\right\}
$$

of the norm $\left\|y\left(t, y_{0}, \varepsilon\right)\right\|$ of solutions of system $\left(1_{(A+Q) / \varepsilon}\right)$, which was obtained in [1] from the Cauchy integral formula [6, p. 166] with the use of the Gronwall-Bellman lemma [6, p. 231] and is valid for any $t \geq 0$; we rewrite this estimate in the form

$$
\left\|y\left(t, y_{0}, \varepsilon\right)\right\| \leq\left\|y_{0}\right\| \exp \left\{\frac{1}{\varepsilon} \int_{0}^{t}[\delta+r(\tau)] d \tau\right\} .
$$

By the assumption of the theorem, there exists a $\delta_{0}>0$ such that $\int_{0}^{t}\left[\delta_{0}+r(\tau)\right] d \tau<0$ for all $t \in\left[t_{0},+\infty\right)$. This, together with the estimate (6), implies that $\left\|y\left(t, y_{0}, t\right)\right\| \rightarrow 0$ as $\varepsilon \rightarrow+0$ at an arbitrary point $t \in\left[t_{0},+\infty\right) \subset(0,+\infty)$ for arbitrary given values $y_{0} \in \mathbb{R}^{n}$ and for any perturbation $Q(t)$ such that $\|Q(t)\| \leq \delta \leq \delta_{0} / 2, t \geq 0$.

Let us show that this convergence is uniform with respect to $t \in\left[t_{0},+\infty\right)$; i.e., for any $\beta>0$, there exists an $\varepsilon(\beta)>0$ such that $\left\|y\left(t, y_{0}, \varepsilon\right)\right\| \leq \beta$ for all $\varepsilon \leq \varepsilon(\beta)$ and $t \in\left[t_{0},+\infty\right)$.

The inequality $\delta \leq \delta_{0} / 2$, together with (2) and (6), implies the estimates

$$
\begin{aligned}
\left\|y_{n}\left(t, y_{0}, \varepsilon\right)\right\| & \leq\left\|y_{0}\right\| \exp \left\{\frac{1}{\varepsilon} \int_{0}^{t}\left[\frac{\delta_{0}}{2}+r(\tau)\right] d \tau\right\} \\
& =\left\|y_{0}\right\| \exp \left\{\frac{1}{\varepsilon}\left[-\frac{\delta_{0}}{2} t+\int_{0}^{t}\left[\delta_{0}+r(\tau)\right] d \tau\right]\right\} \\
& \leq\left\|y_{0}\right\| \exp \left[-\frac{\delta_{0}}{2 \varepsilon} t\right] \leq\left\|y_{0}\right\| \exp \left[-\frac{\delta_{0}}{2 \varepsilon} t_{0}\right]
\end{aligned}
$$

for all $t \in\left[t_{0},+\infty\right)$.

We take an arbitrary number $\beta \in\left(0,\left\|y_{0}\right\|\right)$ and indicate the corresponding value $\varepsilon(\beta)$ independent of $t$ and defined as follows: $\varepsilon(\beta)=\delta_{0} t_{0} /\left[2 \ln \left(\left\|y_{0}\right\| / \beta\right)\right]$. Then the estimate (7) implies the inequality $\left|y\left(t, y_{0}, \varepsilon\right)\right|<\beta$ valid simultaneously for all $\varepsilon \leq \varepsilon(\beta)$ and all $t \in\left[t_{0},+\infty\right)$. Therefore, the solution $y\left(t, y_{0}, \varepsilon\right)$ tends to the zero solution uniformly with respect to $t$ on the entire infinite interval $\left[t_{0},+\infty\right)$ as $\varepsilon \rightarrow+0$. The proof of the theorem is complete.

Remark. From the estimate (7), we obtain the inequality

$$
\left\|y\left(t, y_{0}, \varepsilon\right)\right\| \leq R \exp \left[-(2 \varepsilon)^{-1} \delta_{0} t_{0}\right], \quad t \in\left[t_{0},+\infty\right),
$$

for all vectors $y_{0},\left\|y_{0}\right\| \leq R$, in the $n$-dimensional ball $D_{R}$ with arbitrary given radius $R$ and with center the origin, which implies that for each $\beta \in(0, R)$, there exists an

$$
\varepsilon(\beta)=\delta_{0} t_{0} \times 2^{-1} \ln ^{-1}(R / \beta)
$$

such that for all $\varepsilon \in(0, \varepsilon(\beta)]$, the inequality

$$
\left\|y\left(t, y_{0}, \varepsilon\right)\right\| \leq \beta
$$


is valid simultaneously for all $t \in\left[t_{0},+\infty\right)$ and $y_{0} \in D_{R}$. Therefore, the convergence

$$
\left\|y\left(t, y_{0}, \varepsilon\right)\right\| \rightarrow 0, \quad \varepsilon \rightarrow+0,
$$

claimed in the theorem is uniform with respect to $t \in\left[t_{0},+\infty\right)$ as well as with respect to vectors $y_{0}$ in the ball $D_{R}$.

\section{REFERENCES}

1. Krasovskii, S.G., Differ. Uravn., 1996, vol. 32, no. 2, pp. 277-279.

2. Bylov, B.F., Vinograd, R.E., Grobman, D.M., and Nemytskii, V.V., Teoriya pokazatelei Lyapunova $i$ ee prilozheniya $k$ voprosam ustoichivosti (Theory of Lyapunov Exponents and Its Applications to Stability Problems), Moscow, 1966.

3. Kudryavtsev, L.D., Mat. entsikl. (Mathematical Encyclopedia), Moscow, 1984, vol. 4, p. 786.

4. Fikhtengol'ts, G.M., Kurs differentsial'nogo i integral'nogo ischisleniya (Course of Differential and Integral Calculus), Moscow, 1970, vol. 2.

5. Izobov, N.A., Itogi Nauki Tekh. Ser. Mat. Anal., 1974, vol. 12, pp. 71-146.

6. Andrianova, L.Ya., Vvedenie $v$ teoriyu lineinykh sistem differentsial'nykh uravnenii (Introduction to Theory of Linear Systems of Differential Equations), St. Petersburg, 1992. 